\title{
Is LDL-C level a good indicator of coronary risk in people with nondialysis-dependent CKD?
}

\begin{abstract}
A Canadian study published in the Journal of the American Society of Nephrology reports that LDL cholesterol (LDL-C) level might be a less useful marker of coronary risk in individuals with nondialysis-dependent chronic kidney disease (CKD) than it is in the general population, as the presence of CKD modifies the association between LDL-C level and risk of myocardial infarction.

"In the general population, people with higher cholesterol levels are at increased risk of poor outcomes such as heart attack and stroke, so cholesterol levels are often used to determine who should receive statin treatment, which reduces the risk of these poor outcomes," explains Marcello Tonelli, an author on the latest paper. "Among patients on haemodialysis, LDL-C levels do not reliably identify those at the highest risk of coronary events, but less is known about the association between LDL-C level and coronary risk among patients with nondialysis-dependent CKD. Because current guidelines advise that LDL-C should be used to select people with nondialysis-dependent CKD for lipid-lowering treatment, this issue is clinically important."

Tonelli and co-workers used data from 836,060 adults included in the Alberta Kidney Disease Network database for whom at least one outpatient measurement of serum creatinine taken between 2002 and 2009 was available, as well as at least one measurement of proteinuria and of LDL-C, both obtained within 6 months of the serum creatinine value. The researchers excluded patients with stage $5 \mathrm{CKD}$ (that is, those with an estimated glomerular filtration rate [eGFR] of $<15 \mathrm{ml} / \mathrm{min} / 1.73 \mathrm{~m}^{2}$, kidney transplant recipients and patients on dialysis). They used the Chronic Kidney Disease Epidemiology Collaboration equation and a standardized serum creatinine assay to estimate baseline eGFR
\end{abstract}

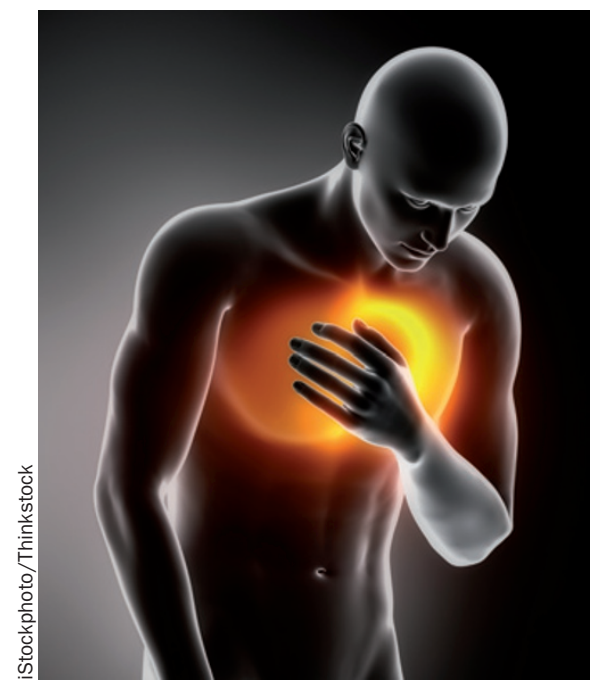

for each individual included in the study, and followed up participants until 31 March 2009. The primary outcome was hospitalization for myocardial infarction.

Over a median follow-up period of 48 months, 7,762 patients were hospitalized for myocardial infarction, with the highest incidence in participants with the lowest eGFRs. Tonelli et al. found that the unadjusted rate of myocardial infarction among patients who were in the lowest eGFR category $\left(15-59 \mathrm{ml} / \mathrm{min} / 1.73 \mathrm{~m}^{2}\right)$ and had the lowest LDL-C levels $(<2.6 \mathrm{mmol} / \mathrm{l})$ was significantly higher than the rate among those who were in the highest eGFR category $\left(\geq 90 \mathrm{ml} / \mathrm{min} / 1.73 \mathrm{~m}^{2}\right)$ and had the highest LDL-C levels ( $\geq 4.9 \mathrm{mmol} / \mathrm{l})$.

Using a model adjusted for factors including baseline age, sex, diabetes, hypertension, proteinuria, statin use and comorbidities, the researchers showed that an LDL-C level $\geq 4.9 \mathrm{mmol} / 1$ (versus a level of $2.6-3.39 \mathrm{mmol} / \mathrm{l}$ ) was associated with the greatest excess risk of myocardial infarction in those in the highest eGFR category. A Cox proportional hazards model showed that the hazard ratios for myocardial infarction associated with each $1 \mathrm{mmol} / \mathrm{l}$ increase in LDL-C level above $2.6 \mathrm{mmol} / \mathrm{l}$ were $1.48,1.33,1.26$, 1.20 and 1.13 for individuals with eGFRs of $90 \mathrm{ml} / \mathrm{min} / 1.73 \mathrm{~m}^{2}, 60 \mathrm{ml} / \mathrm{min} / 1.73 \mathrm{~m}^{2}$, $45 \mathrm{ml} / \mathrm{min} / 1.73 \mathrm{~m}^{2}, 30 \mathrm{ml} / \mathrm{min} / 1.73 \mathrm{~m}^{2}$ and $15 \mathrm{ml} / \mathrm{min} / 1.73 \mathrm{~m}^{2}$, respectively.

"We found that the risk of myocardial infarction was very high in patients with CKD, even when LDL-C level was low," says Tonelli. "To our surprise, higher levels of cholesterol didn't increase this risk very much-it was already high enough to justify treatment. Statins have been shown to reduce clinically important outcomes in people with nondialysisdependent CKD-like those included in our study - who account for $>90 \%$ of CKD patients worldwide. Although patients with CKD are at the highest level of cardiovascular risk, multiple studies show that they are $30-50 \%$ less likely to receive preventive treatments than those with normal kidney function. Our finding suggests that most patients with kidney disease (including those with reduced eGFR, albuminuria, or both) should receive statin treatment regardless of their cholesterol level, as is already the case for most patients with diabetes."

"We are gratified to see that the recently published Canadian lipid guidelines already consider patients with CKD (those with a low eGFR, albuminuria or both) to be in the highest category of cardiovascular risk, and recommend treatment with statins for nearly all CKD patients, regardless of their cholesterol levels. We expect the forthcoming international Kidney Disease: Improving Global Outcomes guidelines for dyslipidaemia management in CKD to take a similar position."

Rebecca Kelsey

Original article Tonelli, M. et al. Association between LDL-C and risk of myocardial infarction in CKD. J. Am. Soc. Nephrol. doi:10.1681/ASN.2012080870 